\title{
« Les OGM face aux nouveaux paradigmes de la biologie "
}

\author{
Analyse du colloque COBINA (11-12 février 2009)
}

Jeanne GROSCLAUDE

Directeur de recherche honoraire de I'INRA ;
Membre correspondant de l'Académie
d'Agriculture de France
$<j e a n n e . g r o s c l a u d e @ o r a n g e . f r>$

\begin{abstract}
The assessment and risk management of GMOs are inherited from the paradigm of molecular biology born in the 1960s and from theories of evolutionary biology dominant in the years that saw the onset of GMOs. But these paradigms have been greatly challenged by recent advances in these disciplines: the dissolution of the concept of gene, the identification of epigenetic phenomena transmitted hereditarily, the discovery of the new continent of microRNAs, the metapopulation theory, the ability to trace gene flow over long distances, imply otherwise consider the properties of a genetically modified organism, whether or not dedicated to the dispersion in the environment. A symposium organized last february in Paris, in the framework of the ANR GMOs was designed to take the measure of these transformations of the paradigms of biology and to let clear how these results force to rethink the impacts of GMOs in the light of the new biology.
\end{abstract}

Key words: GMO assessment, gene flow, gene silencing, epigenetics, system biology, toxicology

lective. Donc un moment rare de coexistence des cultures!

\section{Le déroulé des deux journées}

L'éclairage des sociologues, les flux de gènes, la nouvelle vision de la plasticité des génomes, la biologie des systèmes, les nouvelles méthodologies de caractérisation d'un organisme vivant ou de mesure des échanges biologiques dans l'environnement ont nourri ces journées, remarquablement ponctuées par les temps de synthèse ou de pédagogie des deux « grands témoins »: Charles Auffray (CNRS, Villejuif) et Philippe Baret (Université Catholique de Louvain). Une place prépondérante fut consacrée à l'exposé des nouvelles connaissances et des nouveaux concepts de la biologie : après une entrée en matière épistémologique, les flux de gènes, les avancées sur la structure et le fonctionnement des génomes, les nouvelles voies de la biologie furent présentés par les intervenants successifs. Mais le temps fut laissé largement au questionnement : quelles conséquences pour l'action, et pour la régulation des cultures de plantes transgéniques ? Quelle adaptation des normes de l'évaluation des risques alimentaires? Quelles clarifications pour les citoyens non scientifiques? Quelles tensions persistent?

Une entrée en matière épistémologique

Pierre-Benoit Joly (INRA)

Le conflit entre l'évolution permanente des connaissances scientifiques $d^{\prime} u n e$ part, et la lenteur législative, l'immuabilité du droit $d^{\prime}$ autre part, est source de tensions dans la relation de la science et de la société. En étant optimiste, il ne s'agit que d'un décalage dans le temps. Mais il y a aussi un conflit de stabilité entre les connaissances scientifiques, toujours réfutables, et les normes juridiques qui ne sont pas soumises à contestation.

\section{Christophe Bonneuil (CNRS)}

On a sans doute insuffisamment souligné un aspect de la pensée de Darwin relative à sa vision de l'hérédité et donc de l'amélioration génétique : pour Darwin, à chaque génération il y a nécessité de mettre en œuvre de manière continue la sélection massale (c'est-à-dire le choix, au sein d'une population, des spécimens les plus conformes à l'utilisation souhaitée, et leur utilisation comme reproducteurs); " l'excellence » doit être resélectionnée en permanence. Darwin ne pouvait pas s'appuyer sur l'idée qu'elle est gravée dans le génotype, faute de l'émergence du concept de gène : il faudra attendre Mendel quelques décennies plus tard et la biologie « post-darwinienne ».

Sur le socle mendélien et moléculariste, un siècle plus tard, les utilisateurs de la transgénèse (notamment sur les plantes à l'INRA dans les années 1980) font entièrement confiance à la précision de la greffe de gène qu'ils réalisent, et tablent, au plan de la prédiction des risques, sur l'additivité pure et simple des propriétés de la plante receveuse et de celles portées par le gène introduit.

Au cœur de cette biologie moléculaire est l'idée que le génome est la structure de commande-
${ }^{1}$ Pierre-Benoît Joly, INRA, Anne-Marie Chèvre, INRA et Christophe Bonneuil, CNRS. 
ment de toutes les propriétés, déterminées rigidement par les gènes, alors que le monde darwinien faisait une place à l'instabilité permanente, permettant I'adaptation à l'environnement, et à la notion de fonctionnement en réseau.

\section{Les flux de gènes}

Comprendre par « flux de gènes » les déplacements géographiques de gènes par les gamètes mobiles (cas des plantes, par les pollens ou les graines fécondées) ou par des individus migrants entre populations (cas des animaux, des micro-organismes).

Deux visions opposées des flux de gènes cohabitent : ils sont, dans une vision positive, facteurs d'évolution des « métapopulations ", ou bien, dans une vision négative, ils sont facteurs de « contamination », notamment dans le cas du transport de transgènes.

\section{Denis Couvet (MNHN) :}

"L'évolution des conceptions sur le rôle évolutif et les incidences écologiques des flux de gènes " Les flux géniques entre populations d'une même espèce apportent un avantage : diminuer la consanguinité d'une population (exemple : le puma de Floride restauré par le puma du Texas par l'arrivée de $20 \%$ de migrants), mais aussi des inconvénients : frein pour les adaptations locales ou la colonisation de nouveaux biotopes marginaux.

Les flux migratoires influencent les systèmes génétiques, la viabilité vue au niveau de la métapopulation $^{2}$ : risque de perte des migrants dans la matrice environnementale, ou au contraire d'invasion biologique par un migrant donné. Quant à savoir si les migrants quittant une population sont un lot aléatoire, ou des individus "ratés", ou des "conquérants dominants », cela dépend des situations biologiques.

La qualité de la matrice va être déterminante pour autoriser les échanges : c'est tout l'enjeu de bien définir la future trame verte.

Une méthodologie doit se développer afin de mesurer les flux de gènes à l'aide de marqueurs moléculaires et dessiner ainsi une " génétique du paysage » en temps réel, qui ne reflète pas seulement I'histoire ancestrale, mais les flux présents

En conséquence, la génétique des populations doit renouveler ses concepts en distinguant ces arrivées fortuites de gènes selon leur temporalité, leurs effets sur la viabilité des populations (figures 1et 2).

\footnotetext{
${ }^{2}$ Une métapopulation est un groupe de populations de même espèce séparées spatialement mais pouvant entretenir des flux réciproques d'individus.
}

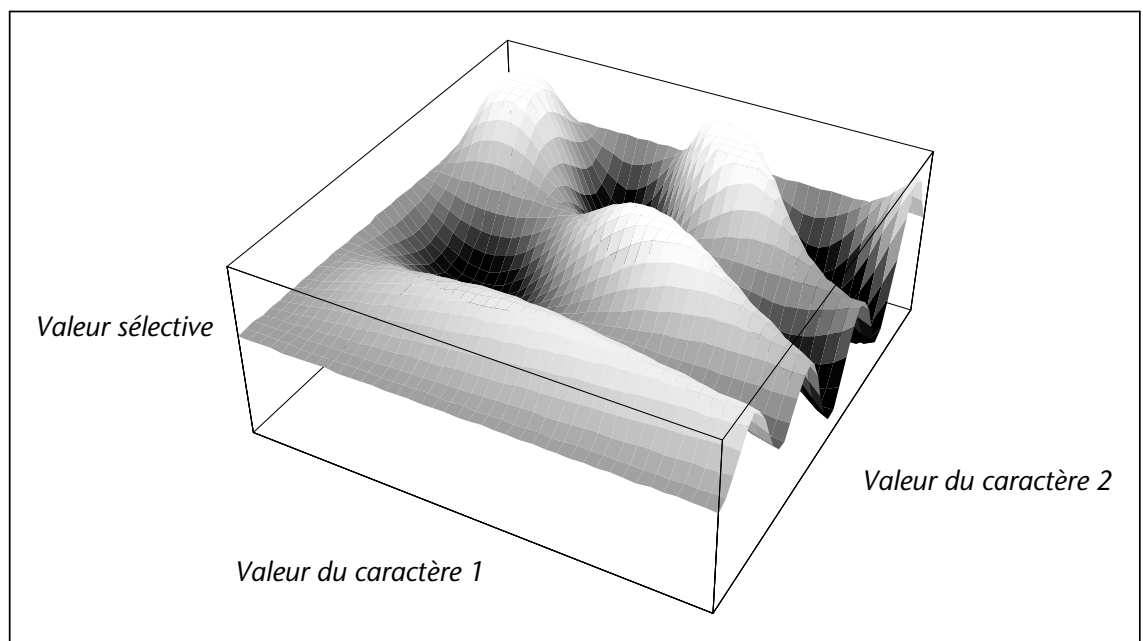

Figure 1. Problématique des paysages adaptatifs (Wright, 1931) : il s'agit de savoir comment la sélection, agissant sur les caractères 1 et 2, permet de passer d'un pic à l'autre, atteindre le pic de valeur sélective maximale.

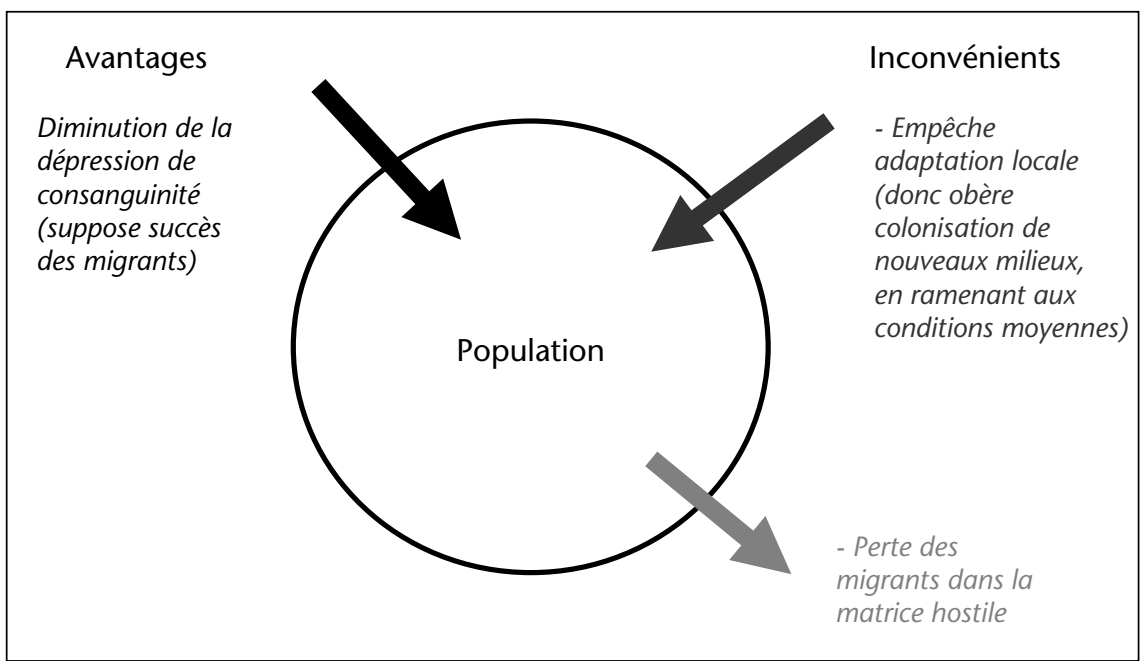

Figure 2. Effets antagonistes du flux génique sur la viabilité de la métapopulation.

Jane Lecomte (Université Paris-Sud) : "Les avancées dans la compréhension des flux de gènes dans les agro-écosystèmes" Première avancée: l'intérêt pour les flux de gènes dans les agro-écosystèmes a été revigoré par la mise en culture des plantes transgéniques, car ces flux doivent être intégrés dans l'évaluation des risques de diffusion du transgène, autant du point de vue écologique qu'agronomique ou économique.

La question des flux de gènes en biologie végétale n'était pas nouvelle et a fait l'objet d'avis contradictoires sur leur rôle dans l'évolution et le comportement démographique des espèces végétales. Des travaux de modélisation intégrant la dimension spatiale de la dispersion du pollen sont apparus dès 1947. Mais ils faisaient peu de place à la fragmentation spatiale des agro-écosystèmes et à leur hétérogénéité, et ne permettaient pas de prédire les événements rares (c'est-à-dire ce qui se passe à des taux faibles de contamination).

Une deuxième avancée va résulter d'un meilleur dialogue entre modélisation et expérimentation et l'évolution de leurs outils respectifs. D'un côté, les protocoles expérimentaux bénéficient de l'utilisation de marqueurs génétiques, d'autre part les données expérimentales sont analysées selon des modèles mécanistes issus de la mécanique des fluides. Enfin, pour prédire le potentiel d'invasion des populations sauvages par les transgènes, les processus de dispersion et les modèles démographiques sont couplés.

Pour aller plus loin, à la fois dans la compréhension des dynamiques de dispersion et dans le pouvoir prédictif des modèles, des progrès doivent être favorisés dans l'acquisition des don- 
nées (observatoires de toutes les communautés de l'agro-écosystème) et le recours à des moyens informatiques conséquents.

\section{Les avancées sur la structure et le fonctionnement des génomes}

Eric Jenczewski (INRA, Versailles) : "La compréhension de la complexité de l'organisation du génome "

Un des moteurs de l'évolution des génomes est la duplication des gènes, phénomène aussi important que l'accumulation de mutations ponctuelles. La polyploïdie, qui est une duplication de l'ensemble du génome, a marqué l'origine de nombreux génomes de plantes: chez Arabidopsis, presque tous les gènes sont dupliqués, signe d'une polyploïdie très ancienne. On retrouve ces duplications dans le génome de l'ensemble des Eucaryotes. Chez I'homme, la variation du nombre de copies rend compte de $0,5 \%$ des divergences entre individus.

La polyploïdisation n'est pas une simple addition des génomes: elle induit de profondes modifications génétiques et épigénétiques, une partie d'un génome peut disparaître ou un transposon peut être activé et ces évolutions peuvent se poursuivre sur un long terme avec des remaniements chromosomiques qui remodèlent le caryotype. L'additivité pure et simple de segments de génome qui juxtaposeraient les propriétés des donneurs n'est donc pas la règle.

\section{Fabien Nogué (INRA, Versailles): « La question de la stabilité du génome des plantes transgéniques à la lumière des travaux récents"}

L'introduction de transgènes, longtemps considérée comme une opération additive précise et contrôlée, déclenche en réalité des modifications inattendues, contredisant la vision de la stabilité des génomes induite par les lois de Mendel et les règles de transmission des caractères. Ces perturbations ne sont pas différentes de celles induites par la mobilité spontanée des transposons, qui affecte d'une part la zone d'insertion dans le génome, $d$ 'autre part le niveau d'expression de l'élément mobile ou introduit artificiellement.

L'insertion d'un transgène perturbe le génome de l'hôte dans la zone d'accueil et peut conduire à l'interruption de l'expression d'un gène de l'hôte (effet mutagène de l'insertion) ou le placer sous commande du promoteur introduit par le transgène, aboutissant à des transcriptions aberrantes; c'est la raison pour laquelle un critère d'évaluation d'une plante transgénique est l'analyse des séquences flanquant le transgène inséré.

L'expression du transgène lui-même est affectée par sa localisation dans le génome et par le nombre de copies insérées, souvent de manière aléatoire dans l'ensemble du génome. On peut aboutir à un silençage du transgène, variable selon la copie considérée. Le mécanisme n'en est pas élucidé.

Pour pallier à ces fortes dérégulations et à l'instabilité des plantes transgéniques qui en résulte, les sélectionneurs doivent pragmatiquement, comme pour les variétés obtenues par d'autres méthodes, vérifier la transmissibilité au cours du temps et après plusieurs générations.

\section{Hervé Vaucheret (INRA, Versailles): "Les nouvelles incertitudes liées aux micro-ARN dans le contexte de la transgenèse "}

La découverte, relativement récente, de l'abondance des petits ARN chez les plantes a ouvert la voie à la compréhension des phénomènes de régulation et de « silencing ${ }^{3}$ » rapide des gènes par «ARN interférence ».

Pratiquement tout le génome est susceptible d'être transcrit en petits ARN, débordant le rôle identifié jusque-là pour les ARN (messagers intermédiaires entre l'ADN et les protéines). Chez Arabidopsis thaliana plusieurs centaines de milliers de petits ARN différents ont été identifiés par séquençage. On distingue les « microARN » (miARN) et les petits ARN interférant (siARN) selon le mécanisme de leur biosynthèse.

La dégradation spécifique des ARN messagers, pour stopper net une fonction cellulaire, est effectuée dans des complexes ribonucléoprotéiques contenant des ARN de petite taille (21 nucléotides) complémentaires des ARN messagers et jouant un rôle d'adaptateur pour charger I'ARN messager à détruire dans le complexe de dégradation.

Ces petits ARN jouent un rôle essentiel dans la stabilité des génomes (contrôle des mouvements des transposons ${ }^{4}$ ), dans la reprogrammation cellulaire qui définit un développement correct et dans la réponse à des stress.

De plus, outre ces petits ARN déduits de ses séquences endogènes, la plante est capable d'activer la synthèse de petits ARN complémentaires des génomes invasifs (à ARN ou à $A D N$ ) issus de pathogènes viraux ou bacté-

\footnotetext{
${ }^{3}$ Le silencing est un phénomène bloquant l'expression d'un gène (le rendant silencieux) sous l'effet de modifications (méthylations) au niveau des histones environnant l'ADN de ce gène, et/ou de dégradation spécifique des ARN produits à partir de cet ADN. Le silencing est un phénomène naturel, intervenant au sein des végétaux et leur permettant de réguler l'expression de leurs gènes ou de lutter contre leurs virus.

${ }^{4}$ Un transposon est une séquence d'ADN capable de se déplacer et de s'insérer par divers mécanismes dans une nouvelle position au sein du génome.
}

riens. Cette machinerie à faire des petits ARN va se « retourner » contre les génomes pathogènes dont ils sont issus et les inactiver, forme la plus primitive de résistance aux pathogènes, retrouvée chez tous les Eucaryotes.

Les transgènes introduits volontairement sont susceptibles d'activer cette même machinerie de « post-transcription silencing » et donc de se faire inactiver rapidement. Le système d'immunité de la plante peut réagir à un transgène comme à un virus et étendre son action à d'autres gènes. II faut donc être très vigilant sur les cascades de modifications indépendantes de l'information portée par le transgène qui peuvent se produire dans une plante transgénique.

Néanmoins ces phénomènes existent dans la nature, et les sélectionneurs classiques, en croisant des génomes par des méthodes naturelles, provoquent les mêmes bouleversements et déclenchements d'incompatibilités: ce sont aussi les petits ARN qui sont à l'œuvre.

La conséquence pour l'évaluation des risques liés à une plante transgénique est que connaître les seules séquences flanquantes du transgène inséré n'est pas suffisant : il faudrait reséquencer toute la plante ! Le seul crible efficace est encore de s'assurer des propriétés fonctionnelles.

Ainsi reconsidérée, la transgenèse perd son caractère d'outil précis capable d'ajouter un caractère prévisisble à une plante; on aura aléatoirement des produits transgéniques n'exprimant pas le même registre de propriétés (figures 3 et 4).

\section{Andras Paldi (Généthon, Evry):}

"Les acquis récents sur l'épigénétique "

Le séquençage complet des génomes ne suffit pas pour comprendre leur régulation. Longtemps on a recherché les mécanismes régulateurs au niveau de séquences intégrées au génome. Or, ces mécanismes spécifiques peuvent être perturbés ou bloqués par l'état de la chromatine, qui joue un rôle majeur dans l'accès aux séquences d'acides nucléiques. La chromatine oscille entre des états stables ou instables : stable elle garantit l'hérédité, instable elle acquiert un potentiel de régulation. Les histones (protéines constituant la chromatine) peuvent être modifiées biochimiquement et réversiblement par des méthylations covalentes grâce à une famille d'enzymes de méthylation ou de déméthylation. Cela conduit à une régulation épigénétique (au niveau de la chromatine) du fonctionnement des gènes.

Peut-on parler d'un "code des histones" coexistant avec le code génétique ? L'hérédité épigénétique peut-elle être expliquée par une combinatoire des modifications biochimiques des acides aminés des histones ? Existe-t-il des "lecteurs» de ce code épigénétique qui seraient eux aussi transmis ? La cartographie de toutes ces modifications peut-elle conduire 


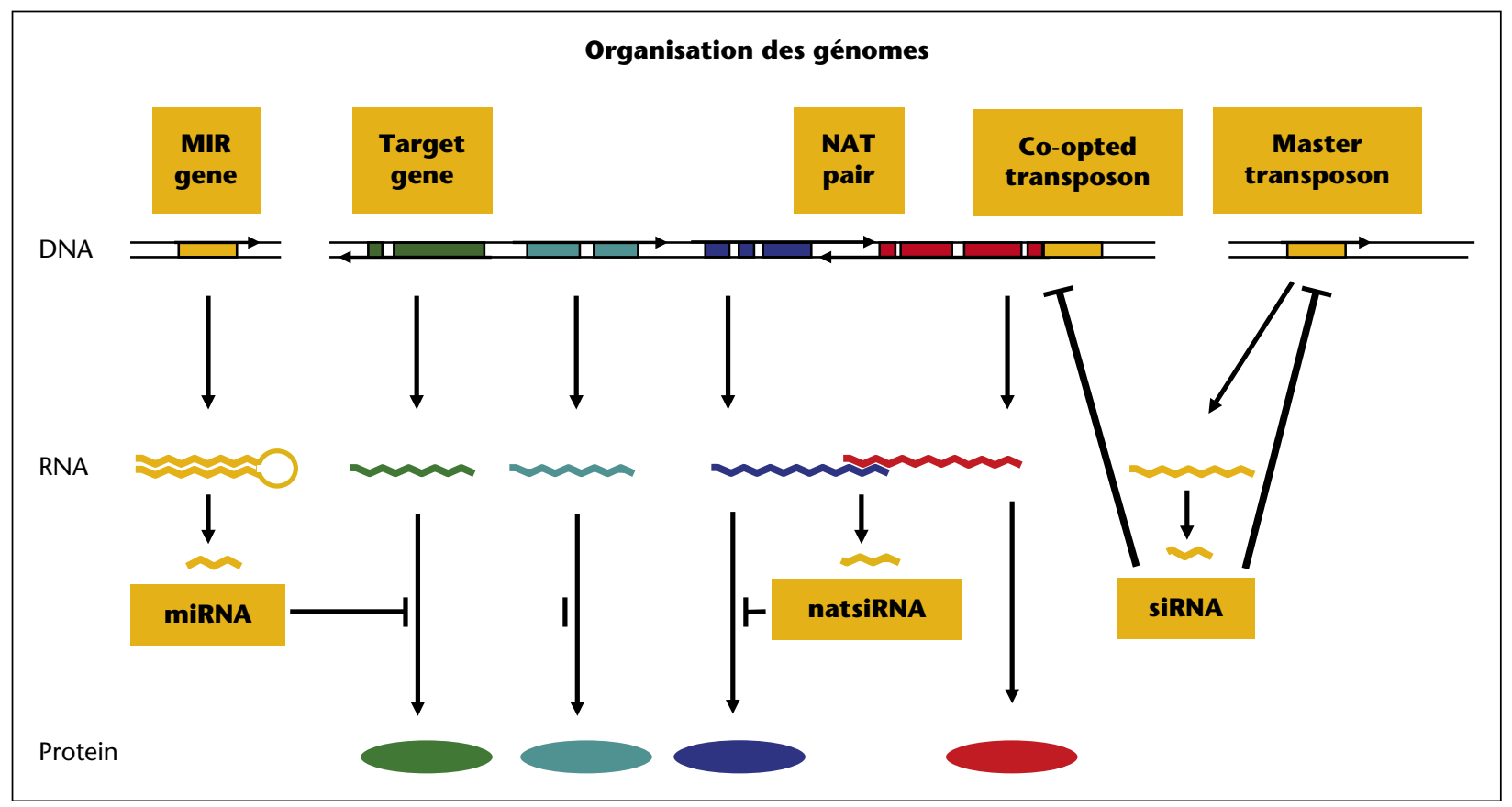

Figure 3. La quasi-totalité de I'ADN génomique des eucaryotes est transcrite en ARN. Les ARNm sont dits "codants » car traduits en protéines (indiquées en vert, turquoise, bleu et rouge sur le schéma). D'autres ARN sont dits non-codants car maturés sous la forme de petits ARN (indiqués en jaune sur le schéma). Les miRNA agissent en trans et régulent au niveau post-transcriptionnel les ARNm qui leur sont complémentaires. Les natsiRNA agissent en cis et régulent au niveau post-transcriptionnel un des deux ARNm dont ils sont issus. Les siRNA agissent en cis et en trans et régulent au niveau transcriptionnel les séquences dont ils sont issus (généralement des transposons) ainsi que les promoteurs de gènes qui ont intégré des bouts de ces transposons. II reste donc peu de place « disponible " pour intégrer des transgènes dans le génome des eucaryotes.

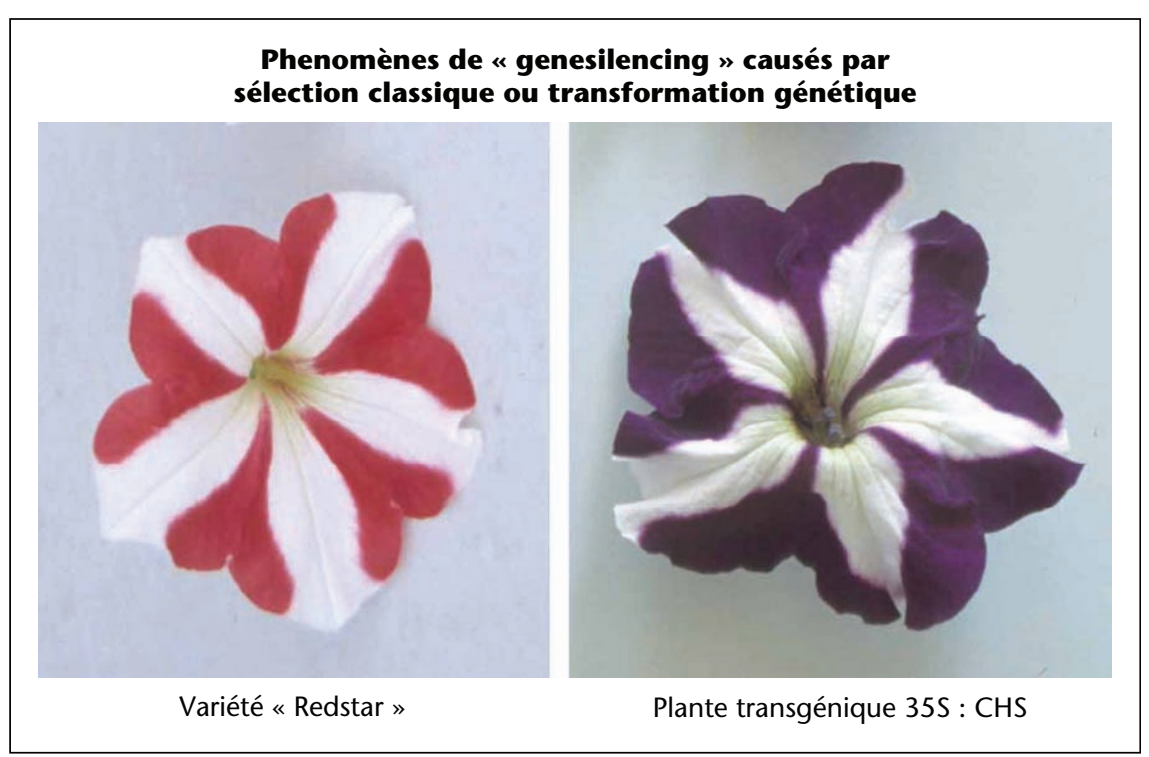

Figure 4. Des modifications de la structure génomique peuvent affecter l'expression des gènes. L'intégration d'un transgène homologue à un gène endogène (ici le transgène 35S:CHS) peut parfois causer l'inactivation (silencing) du transgène mais aussi du gène endogène (l'inactivation du transgène 35S:CHS et du gène CHS endogène est visible au niveau des secteurs blancs des pétales de la fleur de pétunia). Ce phénomène peut aussi être observé lors de croisements entre variétés apparentées. Ainsi, la variéte de pétunia Red star a été obtenue au XVIII siècle en croisant deux variétés de pétunia. Ce croisement a engendré une duplication du gène CHS endogène, causant le même phénotype que celui observé chez des pétunia transgéniques 35S:CHS. À ce jour, on ignore quelle méthode cause le plus de modifications inattendues du génome.

à définir un profil épigénétique ? Quels phénomènes coopératifs s'exercent sur les modifications de la chromatine?
Les fluctuations entre deux états sont au cœur du fonctionnement de beaucoup de systèmes et les théoriciens ont montré que ce « bruit » de fluctuation est essentiel à la pérennité du système. Pourrait-on dire que I'hérédité est plus la transmission de cette dynamique d'oscillation que la transmission d'une caractéristique invariante?

Des observations biologiques vont dans le sens de cette vision de l'hérédité : certains cancers familiaux sont sensibles à des substances qui agissent sur les modifications épigénétiques de la chromatine. Le stress peut canaliser des modifications épigénétiques préexistantes et orienter le phénotype que dicterait le génotype.

En conclusion, sous l'effet de l'environnement, plusieurs phénotypes peuvent correspondre à un même génotype, et suivre une transmission non mendélienne (figures 5 et 6 ).

\section{Les nouvelles voies de la biologie}

François Képès (CNRS, Genopole d'Evry, Programme d'Epigénomique) :

"Les approches complexes en biologie "

Les biologistes, après la phase productive de la collecte d'innombrables données (notamment de séquençage des génomes) par la biologie moléculaire, doivent déchiffrer la complexité de la cellule (compartimentée) et des organismes vivants, en s'adressant à des niveaux croissants d'intégration, où les composantes temporelle et spatiale se surimposentà la seule 


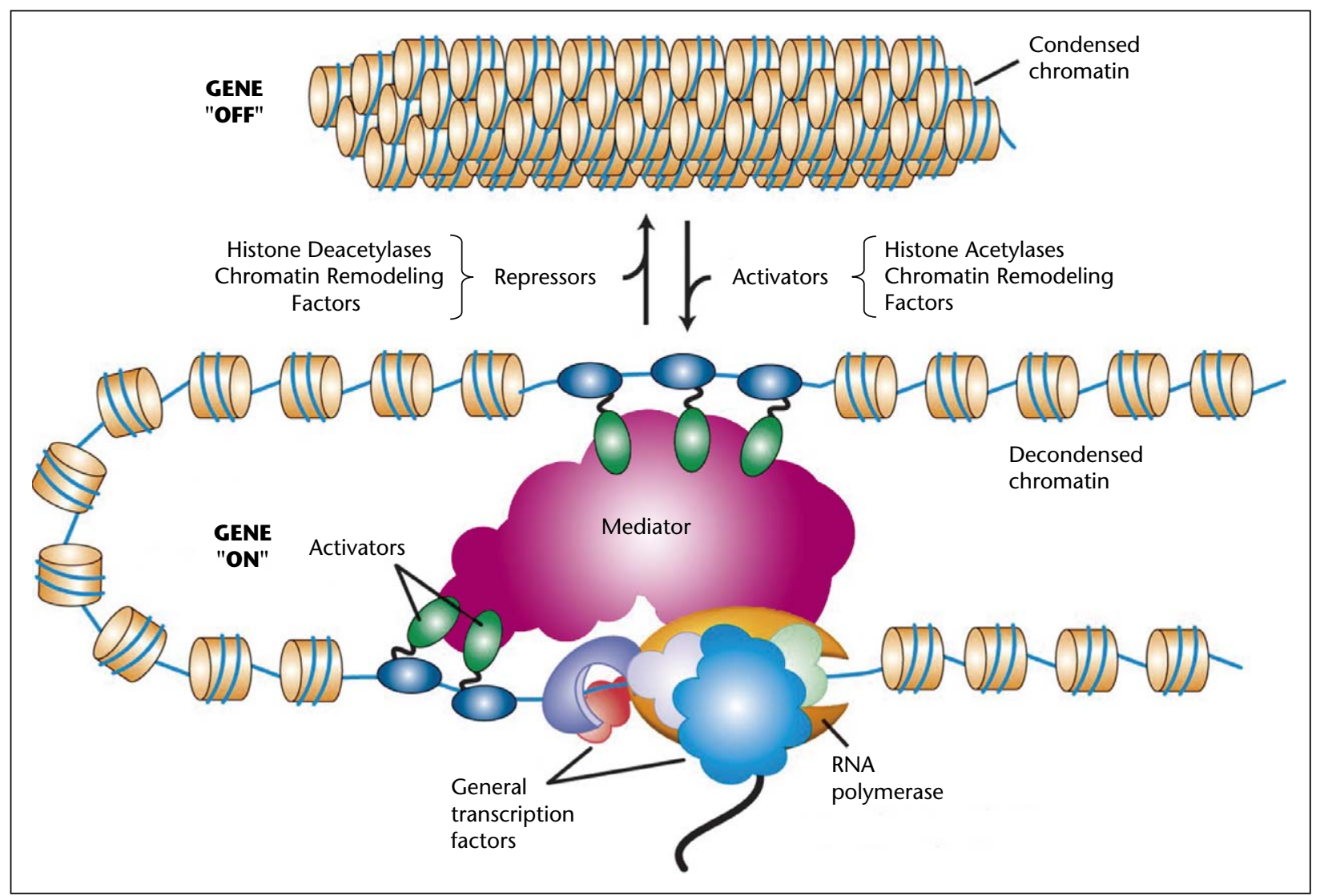

Figure 5. La structure de la chromatine oscille entre un état condensé et un état décondensé sous l'effet de modifications chimiques des acides aminés constitutifs des histones (le rôle des acétylases et des déacétylases est représenté sur ce schéma); il en résulte une régulation de l'activité des gènes par leur degré d'accessibilité pour le complexe de transcription : inactifs sous la chromatine condensée (en haut) et activés lorsque la chromatine est décondensée (en bas).

notion du génome : c'est cette approche qui est désignée par « épigénomique ».

II s'agit de décrire des fonctionnements en réseaux, appuyés sur des modélisations de processus dynamiques, et la hiérarchie des régulations entre ces réseaux.

On touche les limites des capacités de ces modèles: comment distinguer, au niveau $d^{\prime}$ une fonction biologique, un comportement «robuste» et un événement aléatoire (le bruit), alors que les cellules utilisent aussi ce bruit pour diversifier leur phénotype ? La géométrie de la cellule influence les cinétiques de réaction et d'interaction compliquant encore la description des équilibres.

Cette biologie intégrative a besoin de spécialistes non biologistes (des mathématiciens de terrain) pour réaménager la théorie des graphes. Or, la symbiose entre biologistes et spécialistes des réseaux est dissymétrique : les non biologistes se forment "par les livres", alors que les biologistes n'apprennent pas par les livres. Ils ne progressent pas de la même manière : le biologiste a une approche de taupe (il ne peut pas survoler) alors que le mathématicien a une vue cadastrale comme d'un avion.

Pierre Capy (Université Paris-Sud) : « De l'arbre au réseau : l'évolution récente des idées en biologie de l'évolution"

La biologie de l'évolution a intégré la plasticité des génomes, et notamment la part élevée de séquences mobiles à l'intérieur des génomes (les transposons) et des flux de gènes qui passent d'une espèce à l'autre.

Les virus et les bactéries seraient particulièrement actifs dans ces transferts de gènes. Néanmoins, il est difficile de chiffrer la part des rétrotransposons dans l'ensemble des transposons. II est souligné par Jean-Paul Renard que les gènes liés au développement embryonnaire sont majoritairement des retrotransposons.

La représentation en «arbres phylogénétiques » des relations évolutives entre espèces s'appuyait sur les transferts verticaux d'information génétique. Or, si des transferts horizontaux interviennent, ces représentations sont erronées et les arbres linéaires doivent être remplacés par des figurations en réseaux, qui dessinent les convergences évolutives.
Charles Auffray (CNRS, Systemoscope, Villejuif) : « La biologie systémique intégrative : de la molécule à l'écosystème "

La biologie systémique intégrative (BSI) vise une compréhension approfondie du monde vivant à tous les niveaux d'organisation des systèmes biologiques en faisant coopérer plusieurs disciplines scientifiques du vivant et les apports de la théorie des systèmes (mathématiques et informatique).

La BSI s'intéresse aux propriétés émergentes d'un système organisé par comparaison à celles de ses constituants, quelle que soit l'échelle de temps considérée : cela vaut pour l'évolution (ex : réseaux régulateurs des bactéries halophiles et réponse aux changements de leur écosystème), comme pour le développement (ex : le développement embryonnaire vu à travers une approche systémique) ou la physiologie (ex : prédiction de la réponse des patients cancéreux à la chimiothérapie).

Elle vise à développer un cadre conceptuel et méthodologique adapté à une démarche itérative entre expérimentation et modélisation. 


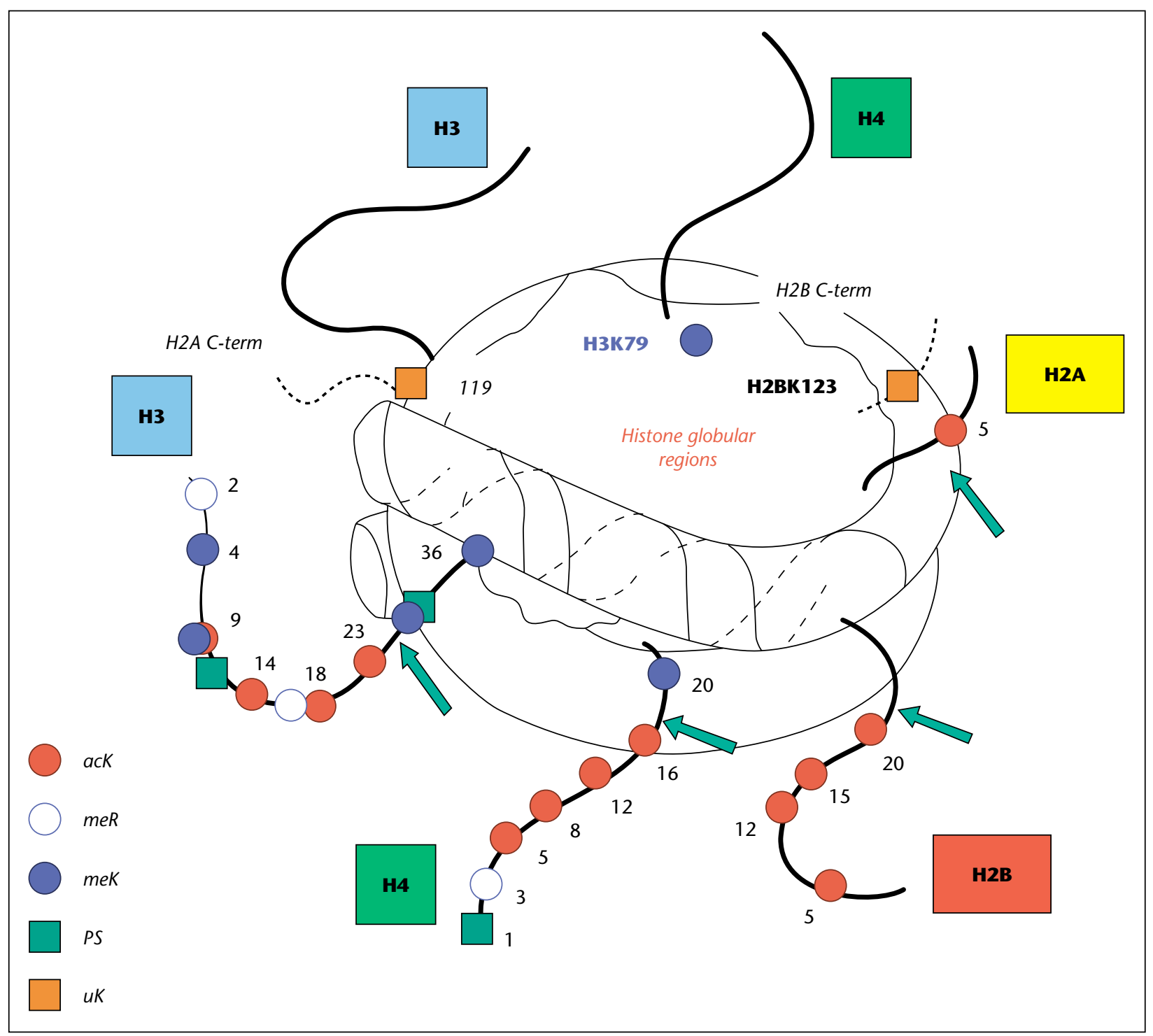

Figure 6. Une cartographie des modifications biochimiques des extrémités exposées des différentes histones $(H 2 A, H 2 B, H 3, H 4)$ entourant un gène : les lysines ( $K)$ et arginines (R) sont modifiées réversiblement par diverses enzymes. La co-occurrence de certaines combinaisons de modifications (marquées par les flèches vertes) et de l'activation/inactivation du gène peut évoquer un « code » épigénétique de régulation de la lecture des gènes, notamment lors de la différenciation cellulaire et du développement embryonnaire.

Partant de questions formalisées (phase 1), on collecte des données sur les composants du système (phase 2), ce qui permet de construire un modèle initial (phase 3); on perturbe le système par le calcul au niveau du modèle, par l'expérimentation dans le système biologique (les outils sont nombreux à ce niveau : ARN interférents, chimie, médicaments, nutrition, environnement) (phase 4); on étudie si le modèle rend compte de ce que l'on observe (phase 5), ce qui permet d'affiner le modèle (phase 6); et on re-exerce des perturbations expérimentales (phase 7), pour réaffiner le modèle (phase 8), etc. Cette approche itérative a été appelée « middle out » pour la distinguer du « bottom up » expérimental et du « top down » de la modélisation.
L'approche systémique minimise la vision du génome qui serait un programme prédéfinissant les propriétés du vivant. L'écologie peut se retrouver dans cette vision !

Les défis de la BSI sont 1) la conception de plans d'expérience intégrant les fluctuations biologiques normales ; 2) la maîtrise des technologies de mesure et de caractérisation ; 3) la maîtrise des méthodes computationnelles et l'intégration des différentes échelles; 4) la capacité à former à ces approches, à favoriser l'inter et la trans-disciplinarité, à trouver les financements appropriés.

Le Systemoscope embrasse l'ensemble de ces défis dans sa devise : "Repenser la recherche, Comprendre le vivant, améliorer la santé ».
Jean-Philippe Cointet (INRA) et Jean-Paul Gaudillère (INSERM) : "Du gène aux réseaux » II suffit, pour comprendre le passage « du code aux connexions ", de comparer la description de l'opéron lactose, dont la régulation est inscrite dans le génome, et le «random network», privilégié pour les interactions protéine/protéine. La propriété émergente, par rapport à ce qui est inscrit dans le génome, est l'architecture qui organise les composants, pas les composants eux-mêmes.

Une autre manière d'évaluer l'évolution de la biologie est d'analyser les co-occurrences de termes dans les publications scientifiques, par exemple celles répertoriées sur PubMed : cela fait apparaître l'émergence de clusters très liés aux technologies nouvelles, de préciser quand 
l'évolution moléculariste s'est installée, ou quand la «post-génomique » a été mobilisée pour I'innovation thérapeutique.

Michel Morange (ENS) : " Le concept de gène à I'heure post-génomique »

La conception du gène a été peu ou prou bousculée par les avancées de la biologie : 1) Même s'il est le conservatoire des molécules du vivant, on sait désormais qu'il est instable. 2) Sa structure ne fait pas l'objet d'une définition unanime: contenu en éléments de régulation, entité chimique?, ce qui n'empêche pas de le manipuler comme un simple fragment $\mathrm{d}^{\prime} \mathrm{ADN}$. 3) La régulation épigénétique s'est imposée, mais elle ne modifie pas les codons et ne prend pas la place de la génétique. Même le mécanisme d'action des petits ARN s'inscrit dans les appariements moléculaires classiques. 4) Sa fonction, historiquement définie par «un gène, une protéine » est plutôt aujourd'hui " un gène, un composant d'un réseau ».

La biologie synthétique n'est pas un simple avatar du génie génétique : on modélise au préalable un module fonctionnel avant d'expérimenter son introduction dans un système vivant.

II s'opère un rapprochement entre la biologie fonctionnelle et la biologie évolutive. Ainsi, pour les plantes génétiquement modifiées, deux communautés tiennent deux discours différents, et pourtant les biologistes de l'évolution se tournent vers les mécanismes moléculaires, tandis que les biologistes fonctionnels intègrent des notions d'évolution pour expliquer la structure et la stabilité des protéines, ou les mutations affectant leur affinité. De fait, I'amélioration des plantes et la biologie moléculaire se rapprochent de l'écologie

\section{Que conclure de ce panorama des nouvelles données biologiques et des nouveaux concepts?}

Au terme de ces présentations, factuelles et conceptuelles, Philippe Baret et Charles Auffray oseront parler de l'émergence d'un para-dogme se substituant aux paradigmes, pour caractériser l'ampleur de l'évolution des concepts en biologie et la rupture avec les anciens dogmes de la biologie moléculariste.

Cette mutation intellectuelle a été provoquée par l'accès à la compréhension des dynamiques, au-delà des simples états descriptifs.

\section{Quelles conséquences}

pour la régulation des cultures de plantes transgéniques?

La réponse de Yves Bertheau (INRA, Versailles) : "Le projet Co-extra et ses implications sur la régulation des OGM "

Ce projet européen multidisciplinaire (associant 200 scientifiques de 18 pays) entend répondre à l'ensemble des problèmes pratiques posés par la coexistence de cultures avec et sans OGM, la traçabilité des OGM et de leurs produits dérivés, les coûts économiques de la coexistence, l'information de tous les opérateurs des filières concernées et des consommateurs.

Ce programme a produit de nouvelles méthodes de détection et de nouvelles stratégies garantissant la séparation des filières avec ou sans OGM, à des seuils de contamination croisée très inférieurs au taux de $0,9 \%$ qui sert actuellement de référence. Le corollaire est de définir (par consensus ?) des aires sans OGM.

\section{La réponse d'Antoine Messéan (INRA,}

Grignon) : "De la modélisation aux politiques publiques de coexistence des cultures"

Même en absence de risque environnemental ou sanitaire certaines productions agricoles revendiquent une absence totale $\mathrm{d}^{\prime} \mathrm{OGM}$. La coexistence entre filières est conditionnée par l'organisation spatiale des cultures, où les flux de gènes sont la principale source de contaminations croisées.

Compte tenu de la grande diversité des contextes à l'échelle des territoires, la modélisation est d'un grand secours pour simuler toutes les situations et les conséquences des scénarios qui pourraient être adoptés.

Ces simulations sont des outils de médiation mais ne prétendent pas se substituer au choix politique.

Les interrogations de Pierre-Benoît Joly (INRA) : "Le gène est-il encore une molécule? Savoirs biologiques et normes de brevetabilité "

L'évolution de la conception du gène soustend les questions autour de sa brevetabilité.

Si on le regarde comme un simple composé chimique (et c'est ce que dit la connaissance bien assise depuis cinquante ans), le débat se centre sur le dilemme: produit de la nature (c'est alors une simple découverte), ou produit manufacturé, sujet à processing (donc brevetable). C'est la vision prédominante qui a inspiré les décisions des années 1970 et 1980 aux USA et entrâné une absence de contestation des brevets sur les séquences d'ADN. Le débat ne sera que technique, et pas politique.

Néanmoins l'exigence que «au moins une fonction de la séquence déposée soit révélée » permet de remonter à une conception plus « mendélienne » (association entre un gène et un caractère souhaité) et moins moléculaire (c'est alors la seule séquence chimique de I'ADN qui fait l'objet du brevet). Cela empêchera le dépôt pour brevet de l'ensemble du séquençage du génome humain réalisé par le programme HUGO. Mais la question d'une autre essence du gène est occultée.
Curieusement, c'est seulement près de vingt ans après le début de la pratique du brevetage des séquences que la contestation va émerger. Dans les années 1990, c'est en Europe qu'est mis en avant le gène comme partie du corps humain, donc non brevetable. La directive européenne 98-44 sanctuarise le génome humain.

Et les tensions entre les différentes conceptions du gène issues de l'évolution de la biologie font irruption dans le débat : il est évident que selon que l'on définit le gène comme une molécule chimique, ou comme une partie du corps humain, ou comme une entité dont l'opérationnalité dépend du contexte épigénétique, on n'évolue plus dans le même cadre de pensée. Au côté bénéfique, pour la progression de la connaissance, de ces incertitudes s'oppose la volonté de réduction de ces objections à des « non-questions » afin d'établir des normes stables et opérationnelles.

Enfin, va se greffer sur le débat « ontologique » et scientifique autour de la définition du gène la remise en cause économique et géopolitique de l'intérêt du brevetage, impliquant beaucoup plus d'acteurs de la société.

\section{Quelle adaptation des normes del'évaluation des risques alimentaires?}

La réponse d'Alain Paris (INRA Toulouse) : " Au-delà de l'équivalence en substance : signature métabolomique

\section{et approches non ciblées "}

De même que l'on a longtemps considéré que la transgenèse n'était que l'addition anodine $d^{\prime} u n$ gène au génome receveur, le principe d'équivalence en substance (introduit dans les années 1990) affirme que la composition d'un végétal GM est équivalente à celle du végétal homologue conventionnel, hormis les substances induites explicitement par le gène introduit. Ce principe était d'autant mieux accepté que la " serendipity ", ou art de trouver ce qu'on ne cherche pas, est plutôt mal accueillie par les spécialistes de la normalisation et du domaine réglementaire.

La recherche de différences ou de présence de métabolites nouveaux, influençant la qualité nutritionnelle ou la sécurité sanitaire des matières premières issues des PGM, était donc la base de la déclaration d'innocuité du produit.

Or, les avancées conceptuelles en biologie des systèmes et en statistiques ont démontré que l'« homéostasie métabolique » n'exclut pas des variations transitoires, des modifications coordonnées en réseau qui brouillent la notion de biomarqueur (une substance particulière qui signalerait une modification significative). C'est ainsi qu'entre cultivars de pommes de terre le maximum de variance se manifeste au niveau de métabolites non identifiés. 
Lorsque l'on veut modifier génétiquement les plantes à vocation alimentaire, on peut induire une rupture complète d'un réseau et créer, non intentionnellement, de nouvelles voies métaboliques, modifiées en flux ou en dispositions topologiques (par exemple, pour les glycoalcaloïdes de la pomme de terre, les teneurs ne sont pas affectées, mais on ne peut pas exclure I'apparition de nouveaux métabolites secondaires, « néobiotiques » éventuellement toxiques). C'est pourquoi, devant l'impossibilité, malgré la puissance des outils d'analyse du métabolome des plantes (spectroscopie de masse et spectroscopie par résonance magnétique nucléaire), d'identifier ces néo-substances et leur éventuelle toxicité, il a été proposé de faire repérer par l'animal les perturbations du métabolisme des plantes.

C'est l'animal, régi lui-même par des lois physiologiques et métaboliques, sa «métabono- mique » propre, qui va réaliser une lecture nutritionnelle de la variation entre cultivars, entre clones (par exemple porteurs du même événement de transgenèse, mais en différents endroits du génome) et révéler ainsi une perturbation toxique.

L'objectivité et l'efficacité de cette démarche sont illustrées par des suivis cinétiques du spectre et du réseau métabolique d'animaux nourris avec différents cultivars, révélant des différences de niveau métabonomique en parfaite corrélation avec les courbes de croissance des différents lots.

Cette démarche combinée, métabolomique sur le végétal, et métabonomique sur l'animal, pourrait permettre de construire de nouvelles références dans l'évaluation comparative des PGM de nouvelle génération et des cultivars plus anciens déjà sur le marché (figure 7).
La réponse du statisticien Marc Lavielle (Université Paris Sud) : "Problèmes statistiques de l'interprétation des essais toxicologiques sur les OGM »

Compte tenu des enjeux sociétaux et des passions qui entourent l'évaluation des OGM, les outils statistiques doivent fournir des résultats fiables appuyés sur de bonnes pratiques statistiques.

Les affirmations contradictoires des scientifiques autour de l'innocuité du maïs MON810 ou de sa toxicité présentent toutes des lacunes méthodologiques: le dossier technique du dossier déposé par Monsanto comme l'étude autrichienne qui a récemment défrayé la chronique. De plus, une observation significative au plan statistique peut n'avoir aucune signification biologique.

C'est sur ce fond contradictoire que la clause de sauvegarde française a été argumentée : il n'y a

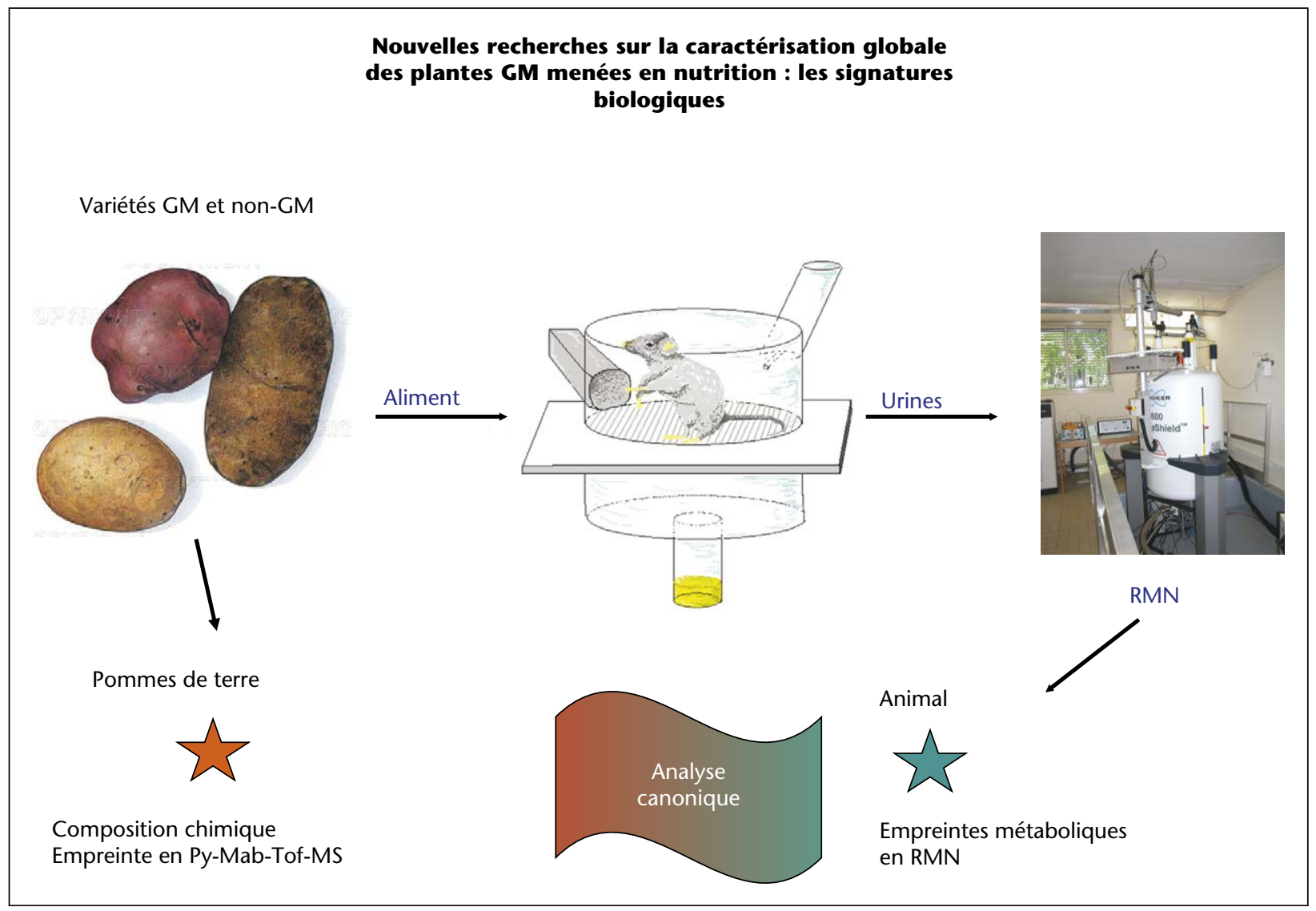

Figure 7. Le principe général sur lequel repose l'évaluation alimentaire des aliments préparés à partir de variétés génétiquement modifiées (GM) est un principe de mise en lien "canonique » entre des données, les plus complètes possibles, produites d'une part par spectrométrie de masse (Py-MAB-Tof-MS) qui sont représentatives de la composition chimique des plantes GM et celles produites par résonance magnétique nucléaire (RMN), d'autre part, sur des biofluides prélevés sur des animaux nourris pendant plusieurs semaines avec ces aliments. Cette « mise en regard " statistique permet de hiérarchiser l'importance des différents facteurs qui influencent la composition chimique des plantes GM, en particulier l'effet cultivar, les effets agronomiques et pédoclimatiques, enfin l'effet lié à la modification génétique de ces cultivars. Des différences dans l'importance relative que ces facteurs peuvent avoir sur la composition et sur la réponse nutritionnelle du modèle peuvent alerter sur la présence d'éventuelles propriétés subtoxicologiques liées à l'événement de transformation des cultivars qui ne seraient pas détectables par la seule analyse de la composition chimique telle qu'elle est mise en ceuvre dans le principe d'équivalence en substances. 
pas de faits nouveaux, mais l'absence de risque n’a pas été établie.

Les normes selon Joël Guillemain (Comité des Biotechnologies, AFSSA) : "Évolution des protocoles et critères de preuve dans les tests toxicologiques "

La toxicologie a pour objectif d'identifier un danger (physique, chimique ou biologique) et d'évaluer le risque créé si des organismes vivants y sont exposés. En toxicologie, particulièrement s'agissant de l'évaluation d'OGM alimentaires, les protocoles doivent s'adapter à l'évolution des sciences d'une part, aux demandes de la société d'autre part.

Parmi ces dernières a émergé une demande de réduction de l'expérimentation animale. Or, les protocoles de détermination de la «DL50» constituaient un vrai massacre d'animaux, et la demande de multiplier les espèces en test aggraverait encore la situation.

Se pose aussi la validité prédictive de tests sur animaux par rapport à l'homme.

La substitution par des analyses "-omiques» $\mathrm{n}$ 'a pas encore atteint une maturation suffisante.

L'administration d'une dose unique maximalisée du produit à tester peut favoriser la manifestation de signes qui n'ont pas de signification biologique. Elle doit être complétée par une administration répétée pendant 90 jours de doses fractionnées, tout en respectant l'équilibre alimentaire de l'animal.

Au plan de l'interprétation, seul un faisceau d'éléments convergents permet de conclure, et en aucun cas un événement isolé ne peut prendre sens. Néanmoins, I'identification de réels biomarqueurs devrait être développée.

Les protocoles doivent être optimisés sans obéir à une attitude de maximisation.

\section{L'accueil par les non-scientifiques de cette immersion dans la science en évolution}

Au cours de ces deux journées, les concepts les plus ardus et les connaissances les plus récentes sur le gène ont été exposés, sans arrogance, par les meilleurs spécialistes devant un certain nombre d'auditeurs peu familiers de ces notions. Comment ceux-ci ont-ils réagi ?
Pour permettre l'expression de la salle, ce sont les auditeurs qui devaient choisir les questions que les participants à la table ronde finale devaient traiter. Inévitablement le débat social sur les OGM est revenu en force, malgré la volonté de centrer ces journées prioritairement sur les évolutions internes à la science.

Plusieurs auditeurs ont apprécié le comportement de chercheurs exposant la déstabilisation progressive des acquis dans leur champ disciplinaire.

Le développement exponentiel des technosciences du vivant a été contesté par un auditeur qui s'est senti « instrumentalisé » par la volonté des organisateurs de rester dans le débat scientifique et de cadrer les débats. C'est le problème de fond de la transmission des connaissances des experts en direction des nonscientifiques, qui subissent l'expérience comme un pouvoir qu'on exerce sur eux. Et qui soupçonnent les scientifiques de ne pas mettre toutes les cartes sur la table.

La participation des citoyens à la science, prônée par certains, est-elle un moyen d'éviter ce sentiment d'être dominé par les porteurs de savoirs?

Le système de formation et la rénovation en cours de la connaissance enseignée, dont témoigne Hervé Vaucheret, peuvent-ils réduire la fracture entre experts et citoyens ?

De fait, la question de l'utilité sociale et des dérives politiques de la maîtrise des OGM s'est imposée à la fin de ces journées dévolues à l'analyse scientifique, en réaction à cette exposition trop parfaite de la connaissance, et ramenant les débats habituels. Un participant à la table ronde a fait part du contraste qu'il a vécu lui-même entre les deux manières de considérer le génome et qui explique l'irréductibilité de ces débats idéologiques : en France, le génome est une sorte de "bibliothèque sacrée » alors qu'aux USA c'est une sorte de coffre-fort où le patrimoine de l'espèce est déposé.

\section{Les protocoles d'évaluation des OGM bougent-ils avec l'évolution de la science?}

L'émergence de nouveaux concepts, du « paradogme » a-t-il déjà produit de nouvelles approches d'évaluation? La réponse est oui, l'expertise a intégré les nouvelles approches de la biologie.

II est suggéré que le principe de précaution pourrait s'appuyer sur la capacité à prédire les propriétés d'OGM même multitransgéniques grâce aux nouveaux modèles théoriques de la biologie des systèmes.

Un intervenant, producteur de PGM, souligne que la part des plantes transgéniques soumises à évaluation est minime par rapport aux stocks d'événements produits, mis «à la poubelle » alors qu'ils contiennent des mines d'information qui permettraient de nouvelles avancées en biologie. Charles Auffray plaide pour la mise en commun de ces connaissances, qu'elles soient d'origine publique ou privée.

Philippe Baret souligne que l'expertise est de plus en plus complexe et appelle un apprentissage, mais souffre de l'idéologisation du débat, de l'élimination de beaucoup d'acteurs et de l'irréversibilité du juridique. II ajoute que les experts sont le plus souvent des fonctionnaires, plus des politiques que des scientifiques, et il doute que des fonctionnaires aient la capacité de faire évoluer le système de l'intérieur.

Un de ces experts fonctionnaires répond que la révision des méthodes d'évaluation est bel et bien engagée, en consultation publique, à I'AFSSA, par sollicitation des instituts de recherche.

\section{Conclusion}

La réussite de ce colloque a été de mettre en scène les évolutions de la biologie qui redessinent l'amélioration génétique et les méthodes d'évaluation des plantes transgéniques, devant un auditoire mêlant au coude à coude experts et non experts.

Au moment où se met en place le Haut Conseil des Biotechnologies, on peut y reconnaître le prototype de réunions d'un nouveau type, exigeantes sur le plan intellectuel, mais permettant aux citoyens de bonne foi et aux chercheurs de bonne volonté de progresser conjointement.

Remerciements. Nous remercions Denis Couvet, Hervé Vaucheret, Andras Paldi et Alain Paris respectivement pour les figures 1-2, 3-4, 5-6 et 7. 